\title{
Diffusion, spin and reaction control in geminate reverse electron transfer
}

\author{
A. I. Burnshtein, ${ }^{* a}$ E. Krissinel $\dagger^{a b}$ and U. E. Steiner ${ }^{a b}$ \\ ${ }^{a}$ Weizmann Institute of Science, Rehovot 76100, Israel \\ ${ }^{b}$ Fachbereich Chemie, Universität Konstanz, 78434 Konstanz, Germany
}

Kinetic analyses of geminate radical escape yields in terms of a simple ("exponential”) reaction scheme with first-order rate constants of separation and geminate recombination have been widely used in the literature, e.g. to evaluate rate constants of reverse electron transfer $\left(k_{- \text {et }}\right)$. Here we demonstrate the limited value of such rate constants by formally analysing, in terms of the exponential model, the diffusion coefficient (viz. viscosity) dependence of the radical escape yield as theoretically calculated in the framework of diffusion-dependent electron transfer theory (unified treatment of non-contact photoinduced forward and geminate reverse electron transfer). It is shown that, while the true electron transfer rate constant is kept constant, the apparent rate constant $k_{- \text {et }}$ from the exponential model undergoes a wide variation as a function of diffusion coefficient and the rate of spin conversion. Nevertheless, the function $k_{-\mathrm{et}}(D)$ represented in a double log plot for various rates of spin conversion provides a useful map suitable to assign characteristic regions of diffusional, spin and reaction control of the geminate process. As an application to real systems the experimental example of the $\left[\mathrm{Ru}(\mathrm{bpy})_{3}\right]^{2+} /$ methylviologen system is reconsidered. Here a magnetic field effect on the $k_{- \text {et }}(D)$ dependence is useful to corroborate the non-contact formation of the radical pair in the photochemical forward electron transfer reaction.

\section{Introduction}

A photoinduced electron transfer process between an excited electron donor $\mathrm{D}^{*}$ and an electron acceptor $\mathrm{A}$ is roughly described as a two-stage process. ${ }^{1-7}$ The bimolecular reaction

$$
\mathrm{D}^{*}+\mathrm{A} \rightarrow\left[\mathrm{D}^{+} \cdots \mathrm{A}^{-}\right]
$$

is the first stage. It results in the creation of a radical pair (RP) $\left[\mathrm{D}^{+} \ldots \mathrm{A}^{-}\right]$. The second stage comprises a reverse electron transfer reaction (geminate recombination)

$$
\left[\mathrm{D}^{+} \cdots \mathrm{A}^{-}\right] \rightarrow \mathrm{D}+\mathrm{A}
$$

and, in competition with it, a diffusive separation into the free radical ions $\mathrm{D}^{+}$and $\mathrm{A}^{-}$

$$
\left[\mathrm{D}^{+} \cdots \mathrm{A}^{-}\right] \rightarrow \mathrm{D}^{+}+\mathrm{A}^{-}
$$

A fully time-resolved observation of this latter stage is often impeded by the fact that the bimolecular stage is slower. Therefore, the efficiency of separation, $\varphi_{\text {sep }}$, is an important observable for obtaining kinetic information on the geminate stage.

The simplest and most widely used kinetic model to analyse $\varphi_{\text {sep }}$, the so-called exponential model, ${ }^{8}$ employs two firstorder rate constants, $k_{- \text {et }}$ and $k_{\text {sep }}$, to quantify the rates of reverse electron transfer and separation, respectively. The model implies that ions are born in a sphere of radius $\sigma$ and also recombine there with a uniform rate $k_{- \text {et }}$. Alternatively ions can escape the sphere and become free (never come back) with a permanent rate

$$
k_{\text {sep }}=\frac{3 r_{\mathrm{c}} D}{\sigma^{3}\left(\mathrm{e}^{r_{\mathrm{c}} / \sigma}-1\right)}
$$

$\dagger$ On leave from the Institute for Water and Environmental Problems, 656099 Barnaul, Russia. where $D$ is the sum of the diffusion coefficients of the two radicals and $r_{\mathrm{c}}$ is the Onsager length (the distance at which the coulomb interaction between the radicals equals $-k T$ ). Although this expression was obtained as the inverse time of diffusional escape from the sphere where ions were born, it is used as a rate of stochastic jumps outside, so that the devastation of the sphere proceeds exponentially in time. The model acquires its name due to this feature of reaction kinetics which in fact is never realised at least in polar solutions. ${ }^{9}$ Although not realistic, the oversimplified rate equations of the exponential model constitute the formal basis for a quantitative consideration of geminate charge recombination. They were widely used by experimentalists especially for studying the quantum yield of charge separation. ${ }^{10}$ As follows from the solution of these equations, $\varphi_{\text {sep }}$ is expressed as:

$$
\varphi_{\text {sep }}=\frac{k_{\text {sep }}}{k_{\text {sep }}+k_{- \text {et }}}=\frac{1}{1+k_{- \text {et }} / k_{\text {sep }}}
$$

Usually this expression is applied to determine $k_{- \text {et }}$ from the observed $\varphi_{\text {sep }}$.

There is a relationship between the recombination rate of the exponential model and the kinetic rate constant of a diffusional model. It can be established if one assumes that the radicals are born in contact and the reverse electron transfer (recombination) also occurs in contact only. The solution of the corresponding problem in diffusion kinetics is ${ }^{9}$

$$
\varphi_{\text {sep }}=\frac{1}{1+\frac{k_{\mathrm{r}}}{4 \pi r_{\mathrm{c}} D}\left(1-\mathrm{e}^{-r_{\mathrm{c}} / \sigma}\right)}
$$

Here $k_{\mathrm{r}}=k_{\mathrm{c}} \exp \left(r_{\mathrm{c}} / \sigma\right)$ is the kinetic rate constant of bimolecular recombination of the radicals (in the limit of $D \rightarrow \infty$ and with the neglect of any spin effects) while $k_{\mathrm{c}}$ is simply related to the exponential model recombination rate $^{10} \quad k_{\mathrm{c}}=k_{-\mathrm{et}}$ $4 \pi \sigma^{3} / 3$. 
Conceptually, it is useful to note that eqn. (1.3) can also be derived from the Collins-Kimball expression ${ }^{11}$ for the bimolecular rate constant, $k_{\mathrm{CK}}$, of diffusion-assisted bulk recombination of $\mathrm{D}^{+}$and $\mathrm{A}^{-}$:

$$
k_{\mathrm{CK}}=k_{\mathrm{d}} \frac{k_{\mathrm{r}}}{k_{\mathrm{r}}+k_{\mathrm{d}}}
$$

Here $k_{\mathrm{d}}$ is the diffusion-controlled bimolecular rate constant:

$$
k_{\mathrm{d}}=4 \pi D \frac{r_{\mathrm{c}}}{1-\mathrm{e}^{-r_{\mathrm{c}} / \sigma}}
$$

Since the expression for $k_{\mathrm{CK}}$ in eqn. (1.4) can be interpreted as a product of the rate constant $k_{\mathrm{d}}$ of diffusion-controlled formation of a contact radical pair and the probability of its recombination, which is just $\left(1-\varphi_{\text {sep }}\right)$, it follows from eqn. (1.4) that

$$
\varphi_{\text {sep }}=\frac{k_{\mathrm{d}}}{k_{\mathrm{r}}+k_{\mathrm{d}}}=\frac{1}{1+k_{\mathrm{r}} / k_{\mathrm{d}}}
$$

Combining eqns. (1.5) and (1.6) yields eqn. (1.3).

The missing link between eqns. (1.2) and (1.6) is provided by the approach of Eigen ${ }^{12}$ who expressed the equilibrium constant $K_{\mathrm{ip}}$ of formation of contact ion pairs by:

$$
k_{\mathrm{ip}}=\frac{k_{\mathrm{d}}}{k_{\mathrm{sep}}}
$$

By using the approximation ${ }^{13}$

$$
K_{\text {ip }}=\frac{4}{3} \pi \sigma^{3} \mathrm{e}^{r_{\mathrm{c}} / \sigma}
$$

the aforementioned expression (1.1) for $k_{\text {sep }}$ can be obtained.

Hence, in the case of contact reaction for both forward and reverse electron transfer, $\varphi_{\text {sep }}$ can be written as:

$$
\varphi_{\text {sep }}=\frac{1}{1+k_{- \text {et }} \frac{\sigma^{3}}{3 D r_{\mathrm{c}}}\left(\mathrm{e}^{r_{\mathrm{c}} / \sigma}-1\right)}
$$

and the rate constant $k_{- \text {et }}$ of the exponential model can be evaluated as

$$
k_{-\mathrm{et}}=\left(\frac{1}{\varphi_{\mathrm{sep}}}-1\right) \frac{3 D r_{\mathrm{c}}}{\sigma^{3}\left(\mathrm{e}^{r_{\mathrm{c}} / \sigma}-1\right)}
$$

This expression has been widely used to "measure" $k_{- \text {et }}$ through $\varphi_{\text {sep }}$ and to analyse the dependence of $k_{- \text {et }}$ on various parameters such as thermodynamic driving force, ${ }^{14,15}$ ionic strength $^{16}$ and temperature. ${ }^{17}$ There is no doubt, however, that, in general, the geminate reaction scenario is more complex. First, electron transfer is not necessarily a contact process, but may create the radical pair with an initial separation of up to several ångströms, and second, since unpaired spins are involved in the recombination of a radical pair, the rate of reverse electron transfer is controlled by spin selection rules and therefore is coupled to the dynamics of multiplicity changes in the radical pair. The latter situation gives rise to magnetic field and spin polarisation effects and has been extensively characterised, both experimentally and theoretically, in the spin chemical literature. ${ }^{18}$ A unified treatment of non-contact reaction of photoinduced forward and geminate reverse electron transfer has been developed by one of the present authors and co-workers. ${ }^{7,8,19}$ Recently the role of spin effects has also been incorporated into this unified treatment. $^{20,21}$

Since for general chemical thinking the exponential model is a basic paradigm, in this paper we will analyse the theoretical and experimental diffusion and spin dependence of $\varphi_{\text {sep }}$ from the perspective of the exponential model. To this end we will consider the behaviour of the apparent rate constant $k_{- \text {et }}$ of reverse electron transfer assigned from $\varphi_{\text {sep }}$ under the assump- tion of validity of the exponential model. By concentrating on a situation typical of the well-studied reaction of photoexcited ruthenium trisbipyridine $\left(\left[\mathrm{Ru}(\mathrm{bpy})_{3}\right]^{2+}\right)$ with methylviologen $\left(\mathrm{MV}^{2+}\right),{ }^{22}$ we will demonstrate how far a naive interpretation of $k_{- \text {et }}$ [evaluated according to eqn. (1.10)] as the specific rate of reverse electron transfer can go wrong, but also that the dependence of the apparent ("exponential") rate constant $k_{-\mathrm{et}}$ on the diffusion coefficient can be quite useful for assigning characteristic kinetic regimes of the geminate recombination process.

\section{Theoretical predictions}

The following spin-dependent reaction scenario will be considered:

$$
{ }^{3} \mathrm{D}^{*}+\mathrm{A} \rightarrow{ }^{3}\left[\mathrm{D}^{+} \cdots \mathrm{A}^{-}\right] \rightarrow \mathrm{D}^{+}+\mathrm{A}^{-}
$$

$\uparrow \downarrow$

$$
\begin{gathered}
{ }^{1}\left[\mathrm{D}^{+} \cdots \mathrm{A}^{-}\right] \rightarrow \mathrm{D}^{+}+\mathrm{A}^{-} \\
\downarrow \\
{ }^{1}[\mathrm{D} \cdots \mathrm{A}]
\end{gathered}
$$

It assumes that very fast spin conversion follows $\delta$-pulse excitation of excited singlet of electron donor, so that only triplet donor molecules are involved in subsequent bimolecular electron transfer resulting in formation of triplet RPs ${ }^{3}\left[\mathrm{D}^{+} \cdots \mathrm{A}^{-}\right]$. They may be born at a distance somewhat larger than contact. The distribution of these distances will depend on the distance law of the forward electron transfer rate constant and on the diffusion coefficient. For recombination, too, a distance-dependent rate law of electron transfer has to be taken into account. But furthermore, electron transfer will be controlled by a spin selection rule. So, triplet/singlet $(\mathrm{T} / \mathrm{S})$ transitions in the RP become an essential element of the geminate kinetics. In most practical cases, recombination is energetically feasible only for singlet $\mathrm{RPs}^{1}\left[\mathrm{D}^{+} \cdots \mathrm{A}^{-}\right]$. This is the situation that we will consider here.

For $\mathrm{T} / \mathrm{S}$ spin transitions two contributions will be taken into account: incoherent $\mathrm{T} / \mathrm{S}$ relaxation due to the uncorrelated spin relaxation in the two radicals of the pair, and coherent $\mathrm{T} / \mathrm{S}$ transitions due to the time-invariant part of the spin Hamiltonian. Of the coherent contributions we will only consider $\mathrm{T} / \mathrm{S}$ mixing due to the Zeeman Hamiltonian. It arises from different $g$-factors of the two radicals and the T/S mixing frequency is proportional to the external magnetic field $B_{0}$.

For this reaction scenario a unified treatment of both forward and reverse electron transfer has been elaborated and a general numerical solution has been developed. ${ }^{21} \mathrm{An}$ analytical solution has been obtained for the special case where coulombic interaction is negligible $\left(r_{\mathrm{c}} \rightarrow 0\right)$, the RPs are born at some fixed distance apart from contact, they recombine on contact and their spin motion is completely described by the incoherent relaxation process. ${ }^{20,23}$ This analytical solution will be used here to demonstrate the essential consequences if one applies a data analysis formally based on the interpretation of $\varphi_{\text {sep }}$ in terms of the exponential model to a situation where actually non-contact creation of radical pairs and spindependent recombination take place.

The analytical solution is expressed in terms of the following parameters: $\sigma$, the contact radius; $r_{0}$, the radius of RP creation; $D$, the sum of the diffusion coefficients of the two radicals; $k_{\mathrm{r}}$, the purely activation-controlled second-order rate constant of spin-allowed recombination (as would be the measured rate constant in a solvent where $D$ is infinite), and $k_{0}$, the rate constant of spin transitions between levels of $|\Delta m| \leqslant 1$. 
The result is ${ }^{20}$

$$
\varphi_{\text {sep }}=1-\frac{\frac{1}{4} k_{\mathrm{r}}\left[\widetilde{G}_{0}\left(\sigma, r_{0}, 0\right)-\widetilde{G}_{0}\left(\sigma, r_{0}, 4 k_{0}\right)\right]}{1+\frac{3}{4} k_{\mathrm{r}} \widetilde{G}_{0}\left(\sigma, \sigma, 4 k_{0}\right)+\frac{1}{4} k_{\mathrm{r}} \widetilde{G}_{0}(\sigma, \sigma, 0)}
$$

where the function $\tilde{G}_{0}\left(\sigma, r_{0}, s\right)$, representing the Laplace transform of the Green's function of free diffusion, is given by:

$$
\tilde{G}_{0}\left(\sigma, r_{0}, s\right)=\frac{1}{4 \pi r_{0} D} \frac{\exp \left[-\left(r_{0}-\sigma\right) \sqrt{s / D}\right]}{1+\sigma \sqrt{s / D}}
$$

Based on eqn. (2.1) it is easy to obtain the effect of a variation of the diffusion coefficient $D$ on $\varphi_{\text {sep }}$. Then, by using eqn. (1.10), we may calculate the effective value of $k_{- \text {et }}$ that follows from such a realistic diffusional dependence of $\varphi_{\text {sep }}$ if the kinetics are interpreted within the frame of the exponential reaction scheme:

$$
k_{-\mathrm{et}}=\frac{3 D}{\sigma^{2}} \frac{1+x-\mathrm{e}^{-a x}}{4 \frac{r_{0}}{\sigma}\left(\frac{k_{\mathrm{d}}}{k_{\mathrm{r}}}-\frac{\sigma}{4 r_{0}}+1\right)(1+x)-\frac{3 r_{0}}{\sigma} x+\mathrm{e}^{-a x}}
$$

with

$$
\begin{gathered}
k_{\mathrm{d}}=4 \pi \sigma D \\
x=\sqrt{4 k_{0} \sigma^{2} / D} \\
a=\frac{r_{0}}{\sigma}-1
\end{gathered}
$$

Here the quantity $x$ figures as a measure comparing the rate constant of spin equilibration $\left(4 k_{0}\right)$ with the inverse of the characteristic diffusion lifetime $\sigma^{2} / D$ of the radical pair.

It must be stressed that if the exponential model would realistically apply, $k_{- \text {et }}$ as evaluated from $\varphi_{\text {sep }}$ should be independent of $D$.

As stated in the Introduction, the parametrization of our calculation will be done with relevance to the ${ }^{3}\left[\mathrm{Ru}(\mathrm{bpy})_{3}\right]^{2+} / \mathrm{MV}^{2+}$ electron transfer system for which the viscosity and magnetic field dependence of $\varphi_{\text {sep }}$ have been experimentally assessed in detail ${ }^{22}$ and for which the spin dynamics is well accounted for by the approach indicated above. The following parameter values were employed:

$$
\begin{aligned}
& \sigma=10 \AA, \quad r_{0}=10.5 \AA, \quad r_{\mathrm{c}}=0, \\
& k_{\mathrm{r}}=10^{6} \AA^{3} \mathrm{~ns}^{-1}, \quad k_{0}=20 \mathrm{~ns}^{-1}
\end{aligned}
$$

These are similar to those used in our previous theoretical study $^{21}$ analysing the viscosity dependence of the magnetic field effect on $\varphi_{\text {sep }}$. Since in water/acetonitrile/ethylene glycol mixtures at high ionic strength the coulomb interaction is strongly screened, the Onsager radius $r_{\mathrm{c}}$ was set to zero. Whereas in ref. 21 the spin situation was approximated by an effective $\mathrm{T}_{0} / \mathrm{S}$ two-level system, in the present work we explicitly took into account all four spin substates of the RP. Because we eliminated this two-level simplification, it turned out to be necessary to increase $k_{\mathrm{r}}$ by about a factor of 3 in comparison with a previous fitting to restore the order-ofmagnitude agreement with the experimentally determined absolute values of $\varphi_{\text {sep }} \cdot{ }^{24}$ As detailed in ref. 21, the distance $r_{0}$ at which the RP is created is subject to a distribution function that varies with the diffusion coefficient $D$. Using a fixed $r_{0}$ over a broad range of $D$ is a fairly crude approximation but still allows one to retain the general features of the results of a more realistic calculation as will be shown further below.

Fig. 1(a) shows a double log plot of $k_{- \text {et }}$ as a function of the inter-radical diffusion coefficient $D$ according to eqn. (2.3). With the values of $\sigma, r_{0}$ and $k_{\mathrm{r}}$ kept fixed as specified above, the rate constant of spin relaxation, $k_{0}$, was varied in multiples of 10 over several orders of magnitude. It is obvious that

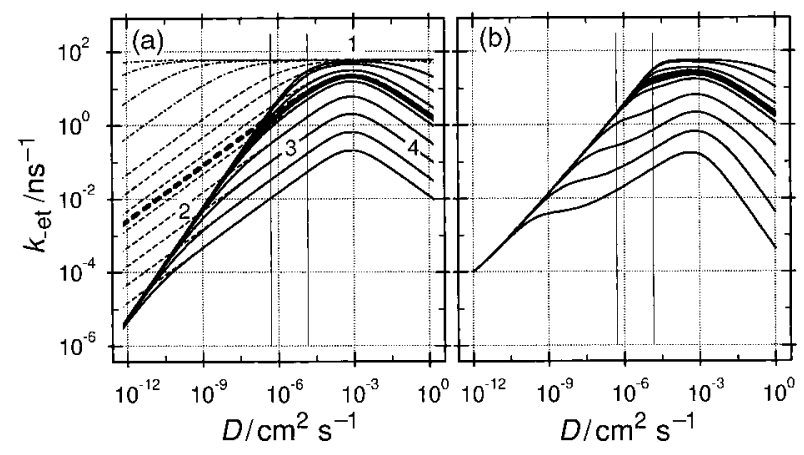

Fig. 1 Apparent (exponential model based) first-order rate constant $k_{- \text {et }}$ of geminate reverse electron transfer as a function of diffusion coefficient $D$ with $\mathrm{T} / \mathrm{S}$ conversion modelled by a rate process with rate constant $k_{0}$. The different lines correspond to different values of $k_{0}$ (from bottom to top, solid and dashed lines: $10^{-3}, 10^{-2}, 10^{-1}, 1,10$ $10^{2}, 10^{3}$ and $10^{4} \mathrm{~ns}^{-1}$; dashed-dotted lines: $10^{6}, 10^{8}, 10^{10}$ and $10^{12}$ $\mathrm{ns}^{-1}$ ). The broad dashed and solid curves combine a set of curves representing the region of $k_{0}$ encountered in the reference experiments (for details see Fig. 3). The experimentally accessible region of $D$ is marked by two vertical lines. (a) Results calculated by eqns. (2.3)-(2.6) with $k_{\mathrm{r}}=10^{6} \AA^{3} \mathrm{~ns}^{-1}$ for RPs recombining in contact. Solid lines are for RPs born with initial separation $r_{0}=10.5 \AA$; dashed lines together with their solid continuations and dashed-dotted lines for RPs born at $r_{0}=\sigma=10 \AA$. (b) Results for RPs whose formation and recombination was treated in the framework of distance-dependent forward and backward electron transfer ( $c f$. ref. 21). The distance dependence of electron transfer was modelled by eqn. (3.1). Other parameters are the same as for the solid curves in part (a)

the constancy of $k_{- \text {et }}$ with $D$, as usually expected when applying a kinetic analysis of $\varphi_{\text {sep }}$ on the basis of the exponential model, only holds for a very limited range of $D$ and at very high values of $k_{0}$. Elsewhere, $k_{- \text {et }}$ may deviate from this limiting behaviour by orders of magnitude. For most cases this fact invalidates the interpretation of $k_{- \text {et }}$ in terms of electron transfer rate only. Nevertheless, the diagram shown in Fig. 1(a) is of considerable heuristic value: the characteristic ranges of $D$ dependence of $k_{- \text {et }}$ may be used to classify four different types of kinetic behaviour associated with specific regions of the $\log \left(k_{- \text {et }}\right) v$ s. $\log (D)$ diagram. These are ( $c f$. assignments in Fig. 1(a)):

1 region of (extended) slope 0

2 region of slope 1

3 region of slope $1 / 2$

4 region of slope $-1 / 2$

Region 1. This region corresponds to the limit $x \rightarrow \infty$, $k_{\mathrm{r}} / k_{\mathrm{d}} \rightarrow 0$. It is actually contracted to a line bounding the possible $\left\{k_{- \text {et }}, D\right\}$ domain from above and has no significant extension in two dimensions. In this border-line case the backward electron transfer is reaction-controlled and this is the only domain where $k_{- \text {et }}$ does not depend on diffusion as it is implied in the exponential model. From eqn. (2.3) one obtains in this limit:

$$
k_{-\mathrm{et},[1]}=\frac{3 k_{\mathrm{r}}}{16 \pi r_{0} \sigma^{2}}=\frac{1}{4} \frac{\sigma}{r_{0}} \frac{k_{\mathrm{r}}}{v}
$$

Here it is indicated that $k_{- \text {et, [1] }}$ can be factorized into three terms: ${ }^{19} k_{\mathrm{r}} / v$, an effective first-order rate constant for spinallowed reaction at contact, obtained from $k_{\mathrm{r}}$ by dividing it by the volume $v=4 \pi \sigma^{3} / 3$ of the reaction sphere [cf. eqn. (1.8)], the factor $\sigma / r_{0}$ corresponding to the probability that after formation at $r=r_{0}$ the RP will eventually encounter at $r=\sigma$; and the factor $1 / 4$ accounting for the spin restriction that only singlet RPs are reactive. 
Region 2. This region corresponds to the limit $x \rightarrow \infty$, $k_{\mathrm{d}} / k_{\mathrm{r}} \rightarrow 0$, i.e. it is the limit of a fully diffusion-controlled backward electron transfer. Like region 1 , region 2 is also a border-line case, but bounding the possible $\left\{k_{- \text {et }}, D\right\}$ domain from the left. From eqn. (2.3) one obtains:

$$
k_{-\mathrm{et},[2]}=\frac{3 D}{\sigma\left(r_{0}-\sigma\right)}
$$

which yields a slope of 1 in the double log plot. When decreasing $D$, the transition from region 1 to region 2 occurs at about

$$
D_{[1,2]}=\frac{k_{\mathrm{r}}}{4 \pi \sigma}\left(1-\frac{\sigma}{r_{0}}\right)
$$

Eqn. (2.8) is only valid for $D \ll D_{[1,2]}$. If $r_{0}$ tends to $\sigma$, i.e. the radical pairs are born at contact, $D_{[1,2]}$ tends to zero and the limiting behaviour of the fully diffusion-controlled type behaviour of backward electron transfer disappears, as would be reasonably expected since for $r_{0}=\sigma$ no diffusion is necessary to bring the radical pair to the recombination distance.

Region 3. This region corresponds to the limit $x \rightarrow 0$, $k_{\mathrm{d}} / k_{\mathrm{r}} \rightarrow 0$, i.e. here the backward electron transfer is diffusionand spin-controlled. The region covers most of the area between the bounding line of limit 2 and the maxima of the $\log \left(k_{- \text {et }}\right)$ vs. $\log (D)$ curves. The limiting form of eqn. (2.3) for this case is:

$$
k_{-\mathrm{et},[3]}=\frac{3}{2} \frac{\sqrt{k_{0} D}}{\sigma}
$$

Consequently, in this region the slope of the curves is $1 / 2$. As pointed out above, region 3 is left-bounded by the line of limit 2. The transition from slope 1 (region 2 ) to slope $1 / 2$ (region 3 ) occurs around

$$
D_{[2,3]}=\frac{1}{4}\left(r_{0}-\sigma\right)^{2} k_{0}
$$

As was mentioned above, for $r_{0} \rightarrow \sigma$ region 3 is actually unbounded on the left, so that for contact-born radical pairs it extends to $D=0$ (cf. dashed lines in Fig. 1).

Region 4. This region corresponds to the limit $x \rightarrow 0$, $k_{\mathrm{r}} / k_{\mathrm{d}} \rightarrow 0$, i.e. here the reaction is reaction- and spin-controlled. As can be seen from the limiting expression derived from eqn. (2.3):

$$
k_{- \text {et, [4] }}=\frac{3}{8 \pi \sigma r_{0}} k_{\mathrm{r}} \sqrt{\frac{k_{0}}{D}}
$$

the slope in the double log plot is $-1 / 2$, i.e. the reaction is inhibited by diffusion. The transition from region 3 to 4 occurs around

$$
D_{[3,4]}=\frac{k_{\mathrm{r}}}{4 \pi r_{0}}
$$

and the transition from region 1 to region 4 is at

$$
D_{[1,4]}=4 \sigma^{2} k_{0}
$$

Thus region 1, i.e. the limiting behaviour of pure reaction control (including the spin statistical factor of $1 / 4$ ) is bounded between $D_{[1,2]}<D<D_{[1,4]}$. If $k_{\mathrm{r}}$ and $k_{0}$ are such that $D_{[1,2]}>D_{[1,4]}$ the plateau region of case 1 does not exist at all.

Creation and recombination in contact. As was pointed out above, the border-line marking region 2 disappears if $r_{0} \rightarrow \sigma$, i.e. if the radical pairs are born in contact. This means that the lines of constant $k_{0}$ in region 3 continue with slope $1 / 2$ all the way down to $\log D \rightarrow-\infty$. This behaviour is indicated by the dashed lines in Fig. 1. The analytical expression obtained in the limit $r_{0} \rightarrow \sigma$ from eqn. (2.3) is

$$
k_{- \text {et }}=\frac{3 D}{4 \sigma^{2}} \frac{x}{\frac{k_{\mathrm{d}}}{k_{\mathrm{r}}} x+\frac{k_{\mathrm{d}}}{k_{\mathrm{r}}}+1}
$$

Actually, this limiting formula for $k_{- \text {et }}$ is completely sufficient to reproduce the curves of Fig. 1 correctly in regions 1,3 and 4 , i.e. only the existence of boundary 2 is a specific feature of non-contact formation of the radical pair. The fact that regions 3 and 4 exist even for the case of combined contact formation and contact recombination of the radical pair indicates that, even in this situation, which is so close to the idea of the exponential model, the constancy of the formally evaluated quantity $k_{- \text {et }}$ with respect to a variation of $D$ is not warranted because of the involvement of spin dynamics in the backward electron transfer.

Distance-dependent forward and backward electron transfer. It has been indicated above that assessing a fixed distance of radical pair creation and recombination over a wide range of $D$-values is an oversimplified picture. Therefore it is instructive to see the corresponding $\log \left(k_{- \text {et }}\right)$ vs. $\log D$ diagram that is obtained if a distance-dependent rate law for both forward and backward electron transfer is taken into account. Details of such a calculation have been described in ref. 21. In Fig. 1(b) we present the results obtained accordingly. The parameters chosen were in accord with those underlying Fig. 1(a) (cf. figure caption). Comparing the two figures we note that the features of the four regions are essentially conserved in the full distance-dependent model, although the values of the slopes in Fig. 1(b) deviate somewhat from those of the analytical model represented by eqn. (2.3). In the transition region between regions 2 and 3 we note a zone with a smaller slope and an inflection point. This latter feature is not borne out in the simplified analytical model represented in Fig. 1(a). The difference exhibited by the two diagrams is essentially due to the difference in $r_{0}$, the distance of RP generation. While $r_{0}$ is an invariable parameter in the simplified model underlying Fig. 1(a), it increases with diminishing $D$ (ref. 21) in the distancedependent model underlying Fig. 1(b). As $r_{0}$ increases the border-line of region 2 is shifted to lower $D$ [cf. eqn. (2.11)]. Thus the curves in region 3 are pulled out to the left and inflection points appear.

\section{Comparison with a real system}

In specifying the fixed parameters $k_{\mathrm{r}}, \sigma$, and $r_{0}$ for the theoretical model described in the last section we referred to the ${ }^{3}\left[\mathrm{Ru}(\mathrm{bpy})_{3}\right]^{2+} / \mathrm{MV}^{2+}$ reaction system, but $D$ and $k_{0}$ were kept varying over a wide range. We will now sharpen our focus on the realistic ranges of the variable parameters $D$ and $k_{0}$ suitable for the system in question. ${ }^{22}$ The $D$ range of experimental interest is indicated by the vertical lines in Fig. 1, the experimentally accessible range of $k_{0}$ values leads to a bunch of curves indicated by the broad curve in the figure. The broadness of this curve corresponds to a variation in $k_{0}$ that is equivalent ( $c f$. below) to the effects that ensue when applying magnetic fields between 0 and $3 \mathrm{~T}$. For reasons of better resolution we will now consider plots with linear scales of $k_{- \text {et }}$ and D. Fig. 2(a) shows the values of $\varphi_{\text {sep }}$ previously observed for a variation of $D$ through solvent viscosity at various values of the external magnetic field. ${ }^{22}$ Fig. 2(b) shows the $k_{- \text {et }}$ values obtained by eqn. (1.10), i.e. from the analysis of $\varphi_{\text {sep }}$ in terms of the exponential model. The relative magnetic field effects on $\varphi_{\text {sep }}$ and $k_{- \text {et }}$ are plotted in Figs. 2(c) and (d). The $D$-dependent magnetic field effect on $\varphi_{\text {sep }}$ has been 

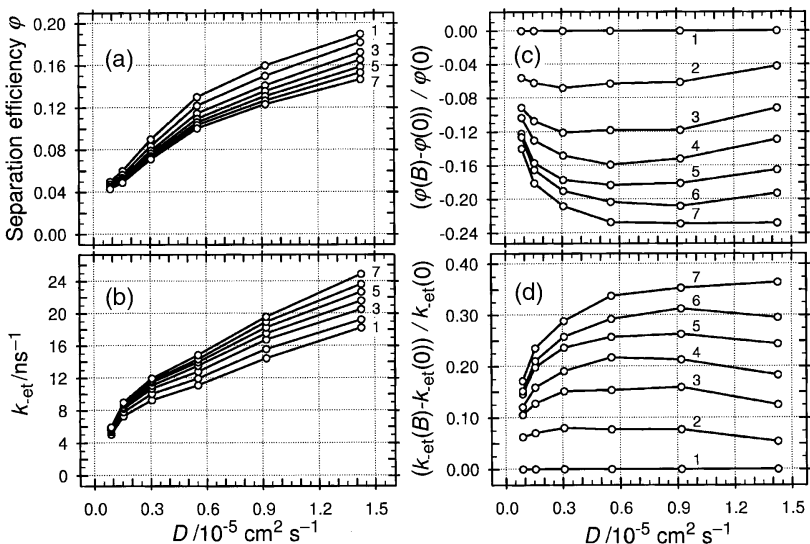

Fig. 2 Experimental data for the ${ }^{3}\left[\mathrm{Ru}(\mathrm{bpy})_{3}\right]^{2+} / \mathrm{MV}^{2+}$ reaction system. ${ }^{22}$ (a) Quantum yields of radical separation $\varphi_{\text {sep }}$ (circles). Curves 1-7 correspond to magnetic fields $B$ of $0,0.5,1.0,1.5,2.0,2.5$ and $3.0 \mathrm{~T}$, respectively. (b) Apparent first-order rate constant of $k_{-}$ of reverse electron transfer as evaluated from the $\varphi_{\operatorname{sep}}$ data in terms of the exponential model eqn. (1.10)]. (c) Relative magnetic field effect on $\varphi_{\text {sep }}$ based on the data in part (a). (d) Relative magnetic field effect on $k_{- \text {et }}$ based on the data in part (b).

analysed in ref. 21 . Here we will concentrate on the $D$ dependence of $k_{- \text {et }}$ to see how it fits in to the general classification of kinetic ranges specified in the last section. In order to see this, we plot relevant slices of the diagrams in Figs. 1(a) and (b) with linear scales. The linear plots are shown in Figs. 3(a)(c) for the analytical model with $r_{0}=\sigma$ and with $r_{0}=\sigma+0.5$ $\AA$, and for the model with variable distance dependence, respectively. As can be seen, all of them bear semiquantitative resemblance to the diagrams with the experimental values.

The analytical model leading to eqn. (2.3) for $k_{- \text {et }}$ does not include the spin dynamic effects of an external magnetic field explicitly. We have found, however, that for a numerical calculation with explicit consideration of the spin Liouvillian the resulting function $k_{- \text {et }}(D)$ obtained for a fixed field $B_{0}$ can be precisely matched with a curve for $B_{0}=0$ but the value of $k_{0}$ changed to a certain (higher) value $k_{0}^{\prime}$. For these calculations we employed the technique developed in ref. 21 with the spin Liouvillian from ref. 25 appropriate for the experimental system under consideration. The distance-dependent electron

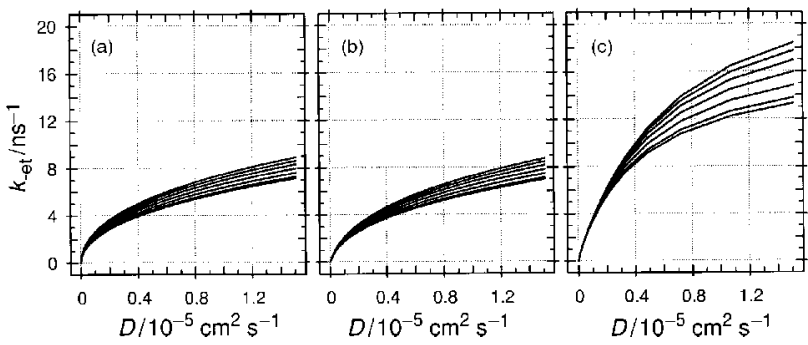

Fig. 3 Linear plot of the first-order rate constant $k_{- \text {et }}$ in the experimentally realized region of $D$ marked in Fig. 1. For the lowest curve in each diagram the value of $k_{0}$ is $20 \mathrm{~ns}^{-1}$. For the other curves the value of $k_{0}$ was chosen such as to represent the kinetic effects of magnetic fields of $0.5,1,1.5,2,2.5$ and $3 \mathrm{~T}$. For details of this assignment see text and Fig. 4. Parameters unless specified below are as in Fig. 1. (a) Contact-born $\left(r_{0}=\sigma=10 \AA\right)$ contact-recombining RPs. $k_{0}=20$, 20.96, 23.18, 25.83, 28.5131 .10 and $33.57 \mathrm{~ns}^{-1}$ (from bottom to top). The lines correspond to the broad dashed curve and its continuation into the broad solid curve in Fig. 1(a). (b) Remotely-born $\left(r_{0}=10.5 \AA\right.$ ) but contact-recombining RPs. $k_{0}=20,21.03,23.42,26.25,29.12,31.88$ and $34.50 \mathrm{~ns}^{-1}$. The lines correspond to the broad solid enhanced curve in Fig. 1(a). (c) RPs with a distribution of creation and recombination distances calculated in the framework of remote forward and backward electron transfer. $k_{0}=20,21.66,25.66,30.51,35.34,39.78$ and $43.70 \mathrm{~ns}^{-1}$. The lines correspond to the broad solid curve in Fig. 1(b). transfer is described by the exponential

$$
W(r)=W_{0} \exp [-(r-\sigma) / L]
$$

where for both forward and backward reactions $W_{0}=10^{3}$ $\mathrm{ns}^{-1}$ and $L=0.75 \AA$, which correspond to the value of $k_{\mathrm{r}}=$ $10^{6}$ also used in the contact approximation. ${ }^{24}$ The fact that it is possible to obtain the same $k_{- \text {et }}(D)$ curve for a calculation with some $k_{0}$ and $B_{0}$ as in a calculation with $B_{0}=0$ but some higher value $k_{0}^{\prime}$ of $k_{0}$ supports the intuitive view that, kinetically, the $\Delta g$-dependent magnetic field effect on the spin dynamics of the RP is to accelerate the conversion between $\mathrm{T}$ and $\mathrm{S}$ states. The empirical relations between $k_{0}^{\prime}$ and $B_{0}$ obtained for the three reaction models considered in this paper are shown in Fig. 4. By using such correlations it is possible to include the magnetic field effect into the analytical model.

From the qualitative comparison of the experimental result in Fig. 2(b) with Figs. 3(a)-(c) we cannot favour one of the theoretical models. A clear distinction arises, however, if we plot the relative magnetic field effects as functions of $D[c f$. Figs. 5(a)-(c)]. Here we see that only the analytical model with distant creation of the radical pair and the model with distance-dependent forward and backward electron transfer reproduce the experimentally observed feature that for $D \rightarrow 0$ the magnetic field effect turns to zero. The contact/contact model behaves differently. Here the magnetic field effect shows a slight increase with decreasing $D$ and reaches a limiting finite value for $D \rightarrow 0$. Thus, the characteristic $D$ dependence of the magnetic field effect on $k_{- \text {et }}$ as exhibited by the experimental results bears evidence of the distant creation of the radical pair in this reaction system. Although, in absolute terms, creation of the radical pair at $10.5 \AA$, i.e. only $0.5 \AA$ wider than "contact", seems quite a subtle difference, the type

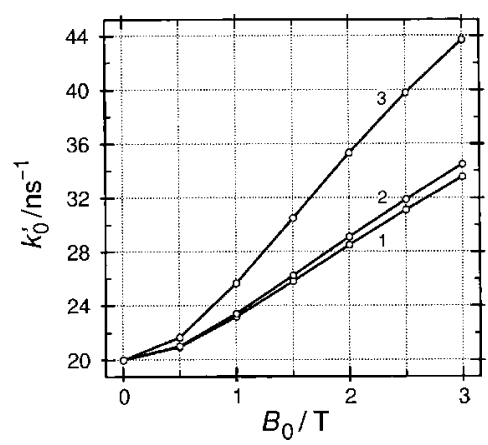

Fig. 4 Empirical equivalence relations between $\mathrm{T} / \mathrm{S}$ transition rate constant $k_{0}^{\prime}$ and magnetic field $B_{0}$ (for details see text). Line 1 corresponds to the case of contact-born and contact-recombining RPs, line 2 to remotely-born and contact-recombining RPs, and line 3 to the case of RPs created and recombining in the course of distancedependent forward and backward electron transfer.

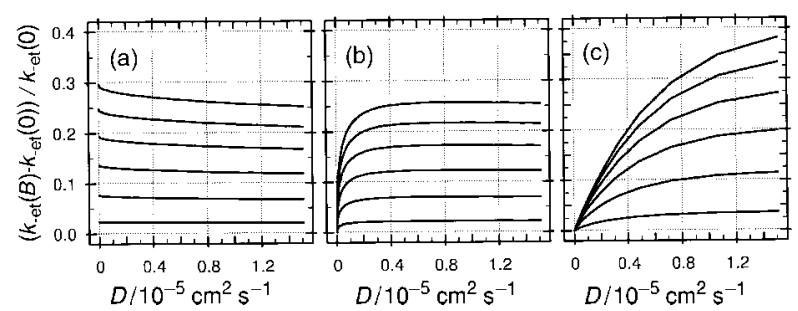

Fig. 5 Relative magnetic field effect on apparent first-order rate constant $k_{- \text {et }}$ theoretically predicted for various models. Linear plots up to the highest experimentally observed $D$ value in the reference system. (a) Contact-born contact-recombining RPs, curves calculated from the curves in Fig. 3(a). (b) Remotely-born contact-recombining RPs, curves calculated from the curves in Fig. 3(b). (c) Formation and recombination of RPs calculated in the framework of remote forward and backward electron transfer [cf. Fig. 3(c)]. 
of $D$ dependence of this magnetic field effect is indeed sensitive enough to make such a definite conclusion.

As seen in Fig. 2(b) the apparent $k_{- \text {et }}$ values obtained from the experiments in the probed $D$ interval range from 5 to 24 $\mathrm{ns}^{-1}$. With these values we should compare the "true" (i.e. $D$-independent) first-order rate constant $k_{\mathrm{r}} / v=240 \mathrm{~ns}^{-1}$ employing the $k_{\mathrm{r}}$ value that follows from the simulation with the diffusion model [cf. Fig. 3(c)] which gives a fair reproduction of the observed $k_{- \text {et }}$ in Fig. 2. Thus it is clearly demonstrated that, apart from an apparently non-realistic $D$ dependence of the quantitiy $k_{- \text {et }}$ evaluated from the exponential model, its value may be much smaller than the value of the "true" first-order rate constant $k_{\mathrm{r}} / v$ of the reverse electron transfer. This is true even if a spin statistical factor of 4 is allowed for.

\section{Conclusions}

It has been shown that the analytically tractable diffusional model with RP creation at non-contact distance $r_{0}>\sigma$, continuous diffusion, first-order kinetic type spin processes and contact recombination Fig. 1(a)] reproduces most of the characteristic features of the numerical solution of a more refined model with a general distance-dependent forward and backward electron transfer and with inclusion of coherent spin processes [Fig. 1(b)]. This should be of practical importance since the analytical result lends itself to a better rationalization, the more so since it allows simple expressions to be derived for the limiting cases of interest.

From what has been presented in this paper it should be clear that the $k_{- \text {et }}$ values evaluated from the radical escape yields by using a spin-independent exponential model are only of limited use as a true measure of the electron transfer rate. From the $D$ dependence of $k_{- \text {et }}$ one can, however, assess which processes control the efficiency of reverse electron transfer.

In particular, we have shown that the $k_{- \text {et }}(D)$ relation is a sensitive indicator of non-contact electron transfer, whenever the kinetic situation approaches the border-line case 2. A still more sensitive indicator of this case is the $D$ dependence of the relative magnetic field effect on $k_{- \text {et }}$ [cf. Figs. 2(d), 3(c), 5(c)]. Thereby, even in moderately viscous solution, a distinction can be made between $r_{0}=\sigma$ (case of RP format at contact) and $r_{0}=\sigma+0.5 \AA$, i.e. differences in creation distance on the order of the length parameter $L$ of the exponential distance law of electron transfer can be detected.

Finally it must be pointed out that, in general, the behaviour $k_{- \text {et }} \rightarrow 0$ for $D \rightarrow 0$ as expressed in Figs. 1 and 2 is characteristic for a situation where the distance of recombination is shorter than the distance of RP formation. In the opposite case, i.e. creation distance shorter than recombination distance, $k_{- \text {et }}$ would increase for $D \rightarrow 0 .{ }^{19}$

\section{Acknowledgements}

Support of this work by the priority programme "Intra- and
Intermolecular Electron Transfer" of the Volkswagenstiftung and Israel Science Foundation is gratefully acknowledged.

\section{References}

1 Schulten, H. Staerk, A. Weller, H.-J. Werner and B. Nickel, $Z$. Phys. Chem. Neue Folge, 1976, 101, 371.

2 N. Mataga, J. Kanda and T. Okada, J. Phys. Chem., 1986, 90, 3880; N. Mataga, T. Asahi, J. Kanda, T. Okada and T. Kakitani, Chem. Phys., 1988, 127, 249; T. Asahi and N. Mataga, J. Phys. Chem., 1989, 93, 6575

3 I. R. Gould, D. Ege, S. L. Matters and S. Farid, J. Am. Chem. Soc., 1987, 109, 3794; I. R. Gould and S. Farid, J. Phys. Chem., 1993, 97, 13067; H. Gan, U. Leinhos, I. R. Gould and D. Whitten, J. Chem. Phys., 1995, 99, 3566.

4 G. Grampp and G. Hetz, Ber. Bunsen-Ges. Phys. Chem., 1992, 96, 198.

5 T. Kakitani, N. Matsuda, A. Yoshimori and N. Mataga, Prog. React. Kinet., 1995, $20,347$.

6 A. I. Burshtein, E. Krissinel and M. S. Mikhelashvili, J. Phys. Chem., 1994, 98, 7319 .

7 A. I. Burshtein and E. Krissinel, J. Phys. Chem., 1996, 100, 3005.

8 K. M. Salikhov, Yu. N. Molin, R. Z. Sagdeev and A. L. Buchachenko, Spin Polarization and Magnetic Effects in Radical Reactions, Elsevier, Amsterdam, 1984.

9 A. I. Burshtein, A. A. Zharikov, N. V. Shokirev, O. B. Spirina and E. B. Krissinel, J. Chem. Phys., 1991, 95, 8013. It should be noted that there is a misprint in eqn. (5.10) to which we refer.

10 A. I. Burshtein, Adv. Chem. Phys., 2000, 114, 419.

11 F. C. Collins and G. G. Kimball, J. Colloid Sci., 1949, 4, 425.

12 M. Eigen, Z. Phys. Chem. Neue Folge, 1954, 1, 176.

13 R. Fuoss, Trans. Faraday Soc., 1934, 30, 967.

14 I. R. Gould, J. E. Moser, D. Ege and S. Farid, J. Am. Chem. Soc., 1988, 110, 1991; I. R. Gould, D. Ege, S. L. Mattes and S. Farid, J. Am. Chem. Soc., 1987, 109, 3794; I. R. Gould, D. Noukakis, L. Gomez-Jah, J. L. Goodman and S. Farid, J. Am. Chem. Soc., 1993, 115, 4405.

15 K. Kikuchi, Y. Takahashi, K. Koike, K. Wakamatsu, H. Ikeda and T. Miyashi, Z. Phys. Chem. Neue Folge, 1990, 167, 27; K. Kikuchi, Y. Takahashi, M. Hoshi, T. Niwa, T. Katagiri and T. Miyashi, J. Phys. Chem., 1991, 95, 2478.

16 M. Z. Hoffman, J. Phys. Chem., 1988, 92, 3458.

17 C. D. Clark and M. Z. Hoffman, J. Phys. Chem., 1996, $100,7526$.

18 See e.g. ref. 8 and the following review: U. E. Steiner and T. Ulrich, Chem. Rev., 1989, 89, 141 and references therein.

19 A. I. Burshtein and N. V. Shokhirev, J. Phys. Chem. A, 1997, 101, 25.

20 A. I. Burshtein and E. Krissinel, J. Phys. Chem. A, 1998, 102, 816.

21 E. B. Krissinel, A. I. Burshtein, N. N. Lukzen and U. E. Steiner, Mol. Phys., 1999, 96, 1083.

22 H.-J. Wolff, D. Bürßner and U. E. Steiner, Pure Appl. Chem., $1995,67,167$

23 Using one rate constant for the overall spin relaxation process corresponds to the situation where spin relaxation of the two radicals in the RP is uncorrelated and $T_{1}=T_{2} \equiv T$ for both radicals $\mathrm{a}$ and $\mathrm{b}$. Then the relation $k_{0}=1 / 4 T_{\mathrm{a}}+1 / 4 T_{\mathrm{b}}$ holds for transitions between any two individual RP spin levels differing by no more than 1 in the spin quantum number $m$.

24 The second-order rate constant $k_{\mathrm{r}}$ is related to the parameter $W_{0}$ in the exponential distance law $W(r)=W_{0} \exp [-(r-\sigma) / L]$ of electron transfer by the relation.

$$
k_{\mathrm{r}}=\int_{0}^{\infty} 4 \pi r^{2} W(r) \mathrm{d} r=4 \pi W_{0}\left(L \sigma^{2}+2 L^{2} \sigma+2 L^{3}\right)
$$

For $L=0.75 \AA$ and $W_{0}=300 \mathrm{~ns}^{-1}$ as used in our previous work, ${ }^{21}$ the corresponding value of $k_{\mathrm{r}}$ would be $0.3 \times 10^{6} \AA^{3}$ $\mathrm{ns}^{-1}$.

25 U. E. Steiner and D. Bürßner, Z. Phys. Chem. Neue Folge, 1990, $169,159$. 